\title{
Polynomial Formulation of Second Derivative Multistep Methods
}

\author{
By S. Kovvali* and G. K. Gupta
}

\begin{abstract}
Following the work of Enright [3] there has been interest in studying second derivative methods for solving stiff ordinary differential equations. Successful implementations of second derivative methods have been reported by Enright [3], Sacks-Davis [9], [10] and Addison [1].

Wallace and Gupta [13] have suggested a polynomial formulation of the usual first-derivative multistep methods. Recently Skeel [11] has shown the equivalence of several formulations of multistep methods. The work of Wallace and Gupta [13] was extended to second derivative methods by Gupta [8]. The present work includes results obtained regarding the stability and truncation error of second derivative methods using the polynomial formulation.
\end{abstract}

1. Introduction. In this paper we extend the work of Wallace and Gupta [13] to include second derivative methods. Gupta [8] presented a polynomial formulation of second derivative methods and we study that formulation further. The present paper also discusses an erroneous result obtained in Gupta [8, Eq. (3.7)]. In addition, the present paper explores the relationship between the blended formulas of Skeel and Kong [12] and the second derivative methods of Enright [3] when Nordsieck representation is used. We also discuss some advantages of the polynomial formulation.

Let the ordinary differential equations being solved be

$$
y^{\prime}=f(x, y), \quad y(0)=y_{0} \quad(y \text { being a vector }) .
$$

The second derivative linear multistep formulas may be represented as follows using the conventional representation

$$
y_{n+1}=\sum_{i=1}^{k} \alpha_{i} y_{n+1-i}+h \sum_{i=0}^{k} \beta_{i} y_{n+1-i}^{\prime}+h^{2} \sum_{i=0}^{k} \gamma_{i} y_{n+1-i}^{\prime \prime} .
$$

If all $\gamma_{i}$ are zero, then we get the usual linear multistep formulas. Enright [3] studied formulas which have all $\gamma_{i}$ zero except $\gamma_{0}$ and found stiffly-stable formulas up to order 9. Enright [4] presents another set of second derivative formulas which are stiffly stable up to order 7. The first set of Enright's formulas has been implemented by Enright [3], Sacks-Davis [9], [10], and Addison [1].

We first discuss the polynomial formulation in the next section. We then discuss analysis of stability in Section 3 and of truncation error in Section 4 if the

Received October 23, 1979; revised May 6, 1981.

1980 Mathematics Subject Classification. Primary 65L05; Secondary 65D30.

Key words and phrases. Second derivative methods, multistep methods, numerical solution of ordinary differential equations, stiff equations.

*Current address: Department of Electrical Engineering, Kansas State University, Manhattan, Kansas 66506. 
polynomial formulation is used. Section 5 deals with implementation of second derivative formulas using the Nordsieck representation. The following section looks at blended formulas and in Section 7 we present some new second derivative formulas using the polynomial formulation.

2. Polynomial Formulation. Wallace and Gupta [13] present a polynomial formulation of the usual first-derivative multistep methods. This formulation has the advantage that it leads naturally to algorithms using the Nordsieck representation of multistep formulas.

Gupta [8] extended the polynomial formulation of Wallace and Gupta to include second derivative methods. For completeness, we reproduce the example discussed in that paper and, in fact, we discuss it here in somewhat more detail.

Example. The example deals with the following third order formula of Enright [3]:

$$
y_{n+1}=y_{n}+\frac{h}{3}\left(f_{n}+2 f_{n+1}\right)-\frac{h^{2}}{6} f_{n+1}^{\prime} \text {. }
$$

The above formula has been derived by obtaining a polynomial approximation $P_{n+1}(x)$ of degree 3 to the solution such that

$$
\begin{aligned}
P_{n+1}\left(x_{n}\right) & =y_{n}, & P_{n+1}^{\prime}\left(x_{n}\right) & =f_{n}, \\
P_{n+1}^{\prime}\left(x_{n+1}\right) & =f_{n+1}, & P_{n+1}^{\prime \prime}\left(x_{n+1}\right) & =f_{n+1}^{\prime} .
\end{aligned}
$$

We now briefly derive $P_{n+1}(x)$ because this leads to the polynomial formulation we are seeking.

Let

$$
\begin{gathered}
x_{n+1}=0, \quad h=1, \quad \text { and } \\
P_{n+1}(x)=a_{n+1}+b_{n+1} x+c_{n+1} x^{2}+d_{n+1} x^{3} .
\end{gathered}
$$

Applying the conditions (2.2) we get

$$
\begin{aligned}
& a_{n+1}=\frac{1}{3}\left(3 y_{n}+f_{n}+2 f_{n+1}-\frac{1}{2} f_{n+1}^{\prime}\right), \quad b_{n+1}=f_{n+1}, \\
& c_{n+1}=\frac{1}{2} f_{n+1}^{\prime}, \quad d_{n+1}=\frac{1}{3}\left(f_{n}-f_{n+1}+f_{n+1}^{\prime}\right) .
\end{aligned}
$$

Now we can see that

$$
P_{n+1}\left(x=x_{n+1}=0\right)=y_{n+1}=a_{n+1}=\frac{1}{3}\left(3 y_{n}+f_{n}+2 f_{n+1}-\frac{1}{2} f_{n+1}^{\prime}\right),
$$

which is the same as the third order formula (2.1) except that $h=1$.

We may also derive an expression for $P_{n}(x)$ in a similar way and obtain

$$
P_{n}(x)=a_{n}+b_{n}(x+1)+c_{n}(x+1)^{2}+d_{n}(x+1)^{3} \text {, }
$$

where $a_{n}, b_{n}, \ldots$ etc. are the same as $a_{n+1}, b_{n+1}, \ldots$ etc. defined in (2.4) except that $n$ is replaced by $n-1$. We are interested in finding $P_{n+1}(x)-P_{n}(x)$ because this leads to the Nordsieck polynomial representation. Let us say that

$$
P_{n+1}(x)=P_{n}(x)+C_{n+1}(x) \text {. }
$$

Then we get

$$
\begin{aligned}
C_{n+1}(x)= & \left(a_{n+1}-a_{n}-b_{n}-c_{n}-d_{n}\right)+\left(b_{n+1}-b_{n}-2 c_{n}-3 d_{n}\right) x \\
& +\left(c_{n+1}-c_{n}-3 d_{n}\right) x^{2}+\left(d_{n+1}-d_{n}\right) x^{3} \\
= & a_{0, n+1}+a_{1, n+1} x+a_{2, n+1} x^{2}+a_{3, n+1} x^{3} \quad \text { (say), }
\end{aligned}
$$


where

$$
\begin{aligned}
& a_{0, n+1}=y_{n}-y_{n-1}+\frac{1}{3}\left(-2 f_{n-1}-3 f_{n}+2 f_{n+1}-2 f_{n}^{\prime}-\frac{1}{2} f_{n+1}^{\prime}\right), \\
& a_{1, n+1}=-f_{n-1}+f_{n+1}-2 f_{n}^{\prime}, \\
& a_{2, n+1}=\frac{1}{2}\left(-2 f_{n-1}+2 f_{n}-3 f_{n}^{\prime}+f_{n+1}^{\prime}\right), \\
& a_{3, n+1}=\frac{1}{3}\left(-f_{n-1}+2 f_{n}-f_{n+1}-f_{n}^{\prime}+f_{n+1}^{\prime}\right) .
\end{aligned}
$$

We can eliminate $y_{n}-y_{n-1}$ from the above expression for $a_{0, n+1}$ by using (2.1). Now we may rewrite $C_{n+1}(x)$ if we note that

$$
a_{3, n+1}=\frac{1}{3}\left(2 a_{2, n+1}-a_{1, n+1}\right), \quad a_{0, n+1}=\frac{1}{3}\left(2 a_{1, n+1}-a_{2, n+1}\right),
$$

and therefore

$$
C_{n+1}(x)=a_{1, n+1}\left(-\frac{x^{3}}{3}+x+\frac{2}{3}\right)+2 a_{2, n+1}\left(\frac{x^{3}}{3}+\frac{x^{2}}{2}-\frac{1}{6}\right) .
$$

It is easy to check that

$$
C_{n+1}\left(x_{n}\right)=C_{n+1}^{\prime}\left(x_{n}\right)=0 \quad \text { since } x_{n}=-1
$$

Also we note that the above expression for $C_{n+1}(x)$ is equivalent to the representation of Gupta [8, Eq. (2.5)], which defines

$$
p(t)=\frac{2}{3}+t-\frac{1}{3} t^{3}, \quad q(t)=-\frac{1}{6}+\frac{1}{2} t^{2}+\frac{1}{3} t^{3},
$$

where $t=\left(x-x_{n+1}\right) / h$. The polynomials $p$ and $q$ are the same as in (2.6) above because we had assumed $x_{n+1}=0$ and $h=1$ and therefore $t=x$.

The above example shows that the second derivative formulas may be expressed as

$$
P_{n+1}(x)=P_{n}(x)+\delta_{1, n+1} p\left(\left(x-x_{n+1}\right) / h\right)+\delta_{2, n+1} q\left(\left(x-x_{n+1}\right) / h\right),
$$

where $p$ and $q$ are constant polynomials representing the second derivative formula. Polynomials $p$ and $q$ corresponding to formulas of order greater than three of Enright [3] are presented in the Appendix.

The scalars $\delta_{1, n+1}$ and $\delta_{2, n+1}$ are chosen to satisfy the differential equation at $x_{n+1}$ and the second derivative condition. That is

$$
\begin{aligned}
& P_{n+1}^{\prime}\left(x_{n+1}\right)=f\left(x_{n+1}, P_{n+1}\left(x_{n+1}\right)\right), \\
& P_{n+1}^{\prime \prime}\left(x_{n+1}\right)=f^{\prime}\left(x_{n+1}, P_{n+1}\left(x_{n+1}\right)\right) .
\end{aligned}
$$

In [8, Eq. (2.5)] a formulation very similar to (2.8) is used. The only difference is that [8] uses the symbol $\delta_{n+1}$ instead of $\delta_{1, n+1}$ and defines $\delta_{2, n+1}$ of (2.8) above as $u \delta_{1, n+1}$. Comparing Eq. (2.6) with [8, Eq. (2.5)], we obtain

$$
u=\frac{2 a_{2, n+1}}{a_{1, n+1}}=\frac{-2 f_{n-1}+2 f_{n}-3 f_{n}^{\prime}+f_{n+1}^{\prime}}{-f_{n-1}+f_{n+1}-2 f_{n}^{\prime}}
$$

for the third order formula (2.1). The above expression for $u$ does not seem to be equal to $h \partial f / \partial y$ as claimed by Gupta [8, Eq. (3.7)].

We have followed a somewhat tedious approach above to obtain expression (2.6) for $C_{n+1}(x)$. The reason for this was to obtain the expression (2.10) for $u$. An easier approach is possible by observing that

$$
C_{n+1}\left(x_{n}\right)=P_{n+1}\left(x_{n}\right)-P_{n}\left(x_{n}\right)=0
$$


and

$$
C_{n+1}^{\prime}\left(x_{n}\right)=P_{n+1}^{\prime}\left(x_{n}\right)-P_{n}^{\prime}\left(x_{n}\right)=0
$$

and therefore

$$
C_{n+1}(x)=(\text { linear polynomial }) \cdot\left(x-x_{n}\right)^{2}
$$

which may be written as

$$
C_{n+1}\left(x_{n+1}+t h\right)=\delta_{1, n+1} p(t)+\delta_{2, n+1} q(t),
$$

where $p(t)$ and $q(t)$ are independent cubic polynomials satisfying $p(-1)=$ $q(-1)=0$ and $p^{\prime}(-1)=q^{\prime}(-1)=0$. For convenience we require that $p^{\prime}(0)=1$, $p^{\prime \prime}(0)=0$ and $q^{\prime}(0)=0, q^{\prime \prime}(0)=1$, which gives the same polynomials as $(2.7)$ above.

We shall look at some of the implementation details of the second derivative methods in Section 5, but we first consider how the polynomials $p$ and $q$ in (2.8) are related to the coefficients $\alpha_{i}, \beta_{i}$ and $\gamma_{i}$ of the conventional representation (1.2). We do this by analyzing the stability of the second derivative formulas using the formulation (2.8).

3. Analysis of Stability. The stability region associated with the second derivative $k$-step formula (1.2) is defined by the characteristic polynomial

$$
\alpha(r)-h \lambda \beta(r)-(h \lambda)^{2} \gamma(r)=0,
$$

where

$$
\alpha(r)=r^{k}-\sum_{i=1}^{k} \alpha_{i} r^{k-i}, \quad \beta(r)=\sum_{i=0}^{k} \beta_{i} r^{k-i}, \quad \gamma(r)=\sum_{i=0}^{k} \gamma_{i} r^{k-i}
$$

Using the polynomial formulation (2.8), the polynomials $\alpha(r), \beta(r), \gamma(r)$ are not immediately known. Under the hypothesis that (2.8) is the polynomial formulation of some second derivative $k$-step formula (1.2), we will derive an expression for the characteristic polynomial (3.1) in terms of the polynomials $p$ and $q$ of (2.8). We follow an approach similar to that of Wallace and Gupta [13] and Gupta [7] for the usual multistep methods.

In studying the stability we are concerned with the behavior of the numerical solution $y_{n}$ as $n \rightarrow \infty$. If the differential equation $y^{\prime}=\lambda y$ is solved using the second derivative method of order $m$, the approximating polynomial at $x=0$ is given by (assuming constant step-size)

$$
P_{0}(x)=\sum_{i=-\infty}^{0} \delta_{1, i} p\left(\frac{x-x_{i}}{h}\right)+\sum_{i=-\infty}^{0} \delta_{2, i} q\left(\frac{x-x_{i}}{h}\right) .
$$

We need to know $\delta_{1, i}$ and $\delta_{2, i}$ at each step to obtain an expression for $P_{0}(x)$.

Let $h \lambda$ satisfy (3.1) for $|r|>1$. Equation (3.1) now defines $h \lambda$ as an algebraic function of $r$ for $|r|>1$. We have that $y_{n}=r^{n}$ is a numerical solution for $y^{\prime}=\lambda y$. For such a solution the corrections $\delta_{1, n}$ and $\delta_{2, n}$ must also be proportional to $r^{n}$. Due to this proportionality, we may express $\delta_{1, n}$ and $\delta_{2, n}$ as follows:

$$
\delta_{1, n}=K_{1} r^{n} \text { and } \delta_{2, n}=K_{2} r^{n} \text {. }
$$

Substituting in (3.3), we have

$$
P_{0}(x)=\sum_{i=-\infty}^{0} K_{1} r^{i} p\left(\frac{x-x_{i}}{h}\right)+\sum_{i=-\infty}^{0} K_{2} r^{i} q\left(\frac{x-x_{i}}{h}\right) \text {. }
$$


Now let

$$
\begin{aligned}
& p(x)=d_{0}+d_{1} x+d_{2} x^{2}+\cdots+d_{m} x^{m}, \\
& q(x)=e_{0}+e_{1} x+e_{2} x^{2}+\cdots+e_{m} x^{m} .
\end{aligned}
$$

Equation (3.5) now becomes

$$
P_{0}(x)=\sum_{i=-\infty}^{0} K_{1} r^{i} \sum_{j=0}^{m} d_{j}\left(\frac{x-x_{i}}{h}\right)^{j}+\sum_{i=-\infty}^{0} K_{2} r^{i} \sum_{j=0}^{m} e_{j}\left(\frac{x-x_{i}}{h}\right)^{j} .
$$

We now reverse the order of summation and obtain

$$
P_{0}(x)=K_{1} \sum_{j=0}^{m} d_{j} \sum_{i=0}^{\infty} r^{-i}\left(\frac{x}{h}+i\right)^{j}+K_{2} \sum_{j=0}^{m} e_{j} \sum_{i=0}^{\infty} r^{-i}\left(\frac{x}{h}+i\right)^{j}
$$

and therefore

$$
\begin{aligned}
& P_{0}(0)=K_{1} G+K_{2} H \\
& P_{0}^{\prime}(0)=K_{1} R+K_{2} S \\
& P_{0}^{\prime \prime}(0)=K_{1} T+K_{2} U
\end{aligned}
$$

where

$$
\begin{array}{ll}
G=\sum_{j=0}^{m} d_{j} V_{j}(r), & H=\sum_{j=0}^{m} e_{j} V_{j}(r), \\
R=\sum_{j=1}^{m} j d_{j} V_{j-1}(r), & S=\sum_{j=1}^{m} j e_{j} V_{j-1}(r),
\end{array}
$$

and

$$
T=\sum_{j=2}^{m} j(j-1) d_{j} V_{j-2}(r), \quad U=\sum_{j=2}^{m} j(j-1) e_{j} V_{j-2}(r) .
$$

$V_{j}(r)$ is defined in the same way as in [13]

$$
V_{j}(r)=\sum_{i=0}^{m} r^{-i} i^{j}
$$

This may be evaluated from the recurrence relation

$$
V_{j}(r)=\frac{1}{r-1} \sum_{s=0}^{j-1}\left(\begin{array}{l}
j \\
s
\end{array}\right) V_{s}(r) \text { and } V_{0}(r)=\frac{r}{r-1} .
$$

$P_{0}(x)$ is the polynomial approximating the solution at $x=0$ and so satisfies the differential equation and the second derivative condition at $x=0$. That is, for the differential equation $y^{\prime}=\lambda y$, we have

$$
P_{0}^{\prime}(0)=h \lambda P_{0}(0), \quad P_{0}^{\prime \prime}(0)=(h \lambda)^{2} P_{0}(0)=h \lambda P_{0}^{\prime}(0) \text {. }
$$

Substituting for $P_{0}(0), P_{0}^{\prime}(0)$ and $P_{0}^{\prime \prime}(0)$ from (3.9) to (3.13), we have

$$
K_{1} R+K_{2} S=h \lambda\left\{K_{1} G+K_{2} H\right\}, \quad K_{1} T+K_{2} U=(h \lambda)^{2}\left\{K_{1} G+K_{2} H\right\} .
$$

Eliminating $K_{1}$ and $K_{2}$ from the above two equations gives the following:

$$
(S T-U R)+h \lambda(U G-H T)+(h \lambda)^{2}(H R-G S)=0 .
$$


Cancelling the common factor (say $F$ ) and comparing with (3.1) gives

$$
\alpha(r)=\frac{S T-U R}{F}, \quad \beta(r)=\frac{U G-H T}{F}, \quad \gamma(r)=\frac{H R-G S}{F} .
$$

Example. We again consider the example of the third order formula (2.1). The polynomials $p$ and $q$ in (2.7) are represented by vectors

$$
p=\left[\frac{2}{3}, 1,0-\frac{1}{3}\right]^{T}, \quad q=\left[-\frac{1}{6}, 0, \frac{1}{2}, \frac{1}{3}\right]^{T} .
$$

Therefore we have

$$
\begin{array}{ll}
G=\frac{2}{3} \frac{r^{2}\left(r^{2}-2 r-2\right)}{(r-1)^{4}}, & H=\frac{r^{2}\left(-r^{2}+8 r+5\right)}{6(r-1)^{4}}, \\
R=\frac{r^{2}(r-1)(r-3)}{(r-1)^{4}}, & S=\frac{2 r^{2}(r-1)}{(r-1)^{4}} \\
T=-\frac{2 r(r-1)^{2}}{(r-1)^{4}}, & U=\frac{r(r-1)^{2}(r+1)}{(r-1)^{4}}
\end{array}
$$

Substituting these values in (3.16) and eliminating the common factor $F=$ $r^{3} /(r-1)^{4}$ gives the characteristic equation

$$
(h \lambda)^{2}\left(-\frac{r}{6}\right)+h \lambda \frac{(2 r+1)}{3}-(r-1)=0,
$$

which is identical to the characteristic equation of formula (2.1).

We have therefore shown the relationship between polynomial formulation (2.8) and the conventional formula (1.2) of second derivative methods. Before discussing an implementation of second derivative methods we note that when a second derivative method is expressed using formulation (2.8), its truncation error coefficient may also be computed directly using the formula in the next section.

4. Truncation Error. To obtain an expression for the local truncation error coefficient $K_{m+1}$ for a method of order $m$, we follow an approach similar to that of Wallace and Gupta [13] for polynomial representation of the usual multistep methods.

We omit the details of the derivation and present only the resulting formula. Let the corrector polynomials $p$ and $q$ be given by (3.6). The truncation error coefficient $K_{m+1}$ is then given by

$$
K_{m+1}=\frac{a_{0}+a_{1} s_{1}+\cdots+a_{m} s_{m}}{m ! a_{m}},
$$

where $s_{i}$ is the coefficient of $n$ in the summation $\sum_{j=0}^{n-1} j^{i}, a_{i}=d_{i}+Q e_{i} ; d_{i}, e_{i}$ being the coefficients of polynomials $p$ and $q$ and

$$
Q=\frac{d_{1}+2 d_{2} s_{1}+\cdots+m d_{m} s_{m-1}}{e_{1}+2 e_{2} s_{1}+\cdots+m e_{m-1} s_{m-1}} .
$$

(The numbers $s_{i}$ are the Bernoulli numbers. We have $s_{0}=1, s_{1}=-\frac{1}{2}, s_{2}=\frac{1}{6}$, $s_{4}=-\frac{1}{30}$, etc. and $s_{2 n+1}=0, n=1,2, \ldots$ )

5. Implementation. Gupta [8] has discussed the implementation of second derivative methods using the Nordsieck representation. We present further details of the implementation, particularly about the corrector iterations. 
The predictor-corrector algorithm for second derivative methods may be written as

$$
a_{n+1}=A a_{n}+d \delta_{1}+e \delta_{2},
$$

where $d$ and $e$ are vectors of scaled derivatives of polynomials $p\left(\left(x-x_{n+1}\right) / h\right)$ and $q\left(\left(x-x_{n+1}\right) / h\right)$ of $(2.8)$ at $x_{n+1} \cdot a_{n+1}$ and $a_{n}$ are vectors of scaled derivatives of approximating polynomials $P_{n+1}(x)$ and $P_{n}(x)$ of $(2.8)$ at $x_{n+1}$. We note that the above representation is similar to the Nordsieck representation of the usual multistep method as discussed by Gear [5, p. 216].

$\delta_{1}$ and $\delta_{2}$ in the above representation are computed by satisfying the differential equation and the second derivative condition at $x_{n+1}$ as given by (2.9) and must be computed by an iterative scheme. We discuss one possible iterative scheme here.

Let $b=A a_{n}$ and $b_{i}$ be the $i$ th element of $b$. Similarly let $d_{i}$ and $e_{i}$ be the $i$ th elements of $d$ and $e$. In our representation, $d_{2}=e_{1}=0$ and $d_{2}=1, e_{2}=\frac{1}{2}$. Now the conditions (2.9) to compute $\delta_{1}$ and $\delta_{2}$ may be written as

$$
\begin{aligned}
b_{1}+\delta_{1}-h f\left(x_{n+1}, b_{0}+d_{0} \delta_{1}+e_{0} \delta_{2}\right) & =0 \\
b_{2}+\delta_{2}-\frac{h^{2}}{2} J f\left(x_{n+1}, b_{0}+d_{0} \delta_{1}+e_{0} \delta_{2}\right) & =0
\end{aligned}
$$

We have assumed $f^{\prime}=J f$ (if $\partial f / \partial x=0$ ). From the above two equations we get

$$
\delta_{2}=\left(h J b_{1} / 2-b_{2}\right)+h J \delta_{1} / 2 \text {. }
$$

Substituting for $\delta_{2}$ in the first equation of (5.2), we get the following equation:

$$
b_{1}+\delta_{1}-h f\left(x_{n+1}, b_{0}+e_{0}\left(h J b_{1} / 2-b_{2}\right)+\left(d_{0}+h e_{0} J / 2\right) \delta_{1}\right)=0
$$

In the above equation $J$ depends on $\delta_{1}$ and $\delta_{2}$ since $J=J\left(b_{0}+d_{0} \delta_{1}+e_{0} \delta_{2}\right)$. If we are able to solve this equation and obtain $\delta_{1}$, we can compute $\delta_{2}$ using (5.3). To solve (5.4), we need to use the Newton method as modified by Liniger and Willoughby [6] and Enright [3]. We obtain the following iterations

$$
\delta_{1}^{r+1}=\delta_{1}^{r}-\frac{b_{1}+\delta_{1}^{r}-h f^{r}}{I-h J\left(d_{0}+J h e_{0} / 2\right)},
$$

where $\delta_{1}^{r}$ is the $r$ th approximation of $\delta_{1}$ and

$$
f^{r}=f\left(x_{n+1}, b_{0}+e_{0}\left(J^{r} h b_{1} / 2-b_{2}\right)+\left(d_{0}+J^{r} h e_{0} / 2\right) \delta_{1}^{r}\right) .
$$

If we put

$$
W=I-h J d_{0}-h^{2} J^{2} e_{0} / 2
$$

we may rewrite (5.5) as

$$
W\left(\delta_{1}^{r+1}-\delta_{1}^{r}\right)=-b_{1}-\delta_{1}^{r}+h f^{r} .
$$

Note that these iterations are somewhat different than those obtained by Gupta [8]. Once $\delta_{1}$ is computed, we an compute $\delta_{2}$ and obtain vector $a_{n+1}$ of (5.1).

Computing $W$ requires computation of $J^{2}$. Several ways of avoiding this are discussed by Skeel and Kong [12].

6. Blended Multistep Formulas. We now compare our representation with the blended methods approach of [12]. It has been shown by Skeel and Kong [12] that a "blend" of the $(k+1)$ th order Adams-Moulton formula (AMF) and $k$ th order 
Backward Differentiation Formula (BDF) becomes identical to the second derivative formula of [3] of order $k+1$ for the equation $y^{\prime}=\lambda y$.

In the Nordsieck notation the blended formula is given by

$$
a_{n}=A a_{n-1}+\left(w-h \gamma J_{n} z\right) \Delta_{n},
$$

where $a_{n}, a_{n-1}$ are vectors of scaled derivatives of the approximating polynomials, $w$ is the correction vector corresponding to AMF of order $k+1$, and $z$ to BDF of order $k$.

In polynomial notation the above formula is given by

$$
P_{n}(x)=P_{n-1}(x)+\left(A(t)-\gamma h J_{n} B(t)\right) \Delta_{n},
$$

where $A$ and $B$ are modifier polynomials and $t=\left(x-x_{n}\right) / h$. Comparing the above equation with Eq. (2.8), it would be expected that if (6.2) is to be equivalent to Enright's formulas for some $\gamma$, then we should have

$$
P_{n}\left(x_{n-1}\right)=P_{n-1}\left(x_{n-1}\right)=y_{n-1} \text { and } P_{n}^{\prime}\left(x_{n-1}\right)=P_{n-1}^{\prime}\left(x_{n-1}\right)=f_{n-1} .
$$

Therefore we should have

$$
A(-1)=B(-1)=0 \text { and } A^{\prime}(-1)=B^{\prime}(-1)=0 .
$$

In the blended formulas we note that the condition $B^{\prime}(-1)=0$ is not satisfied, that is, $P_{n}^{\prime}\left(x_{n-1}\right) \neq P_{n-1}^{\prime}\left(x_{n-1}\right)$. Another difference between the Nordsieck representation (6.1) of blended formulas of order $m$ and (5.1) of second derivative formulas of order $m$ is that (6.1) is an $m$-value method based on $(m-1)$ degree polynomials while (5.1) is an $(m+1)$-value method based on $m$ degree polynomials.

It is therefore not obvious that the blended formulas are equivalent to Enright's formulas for $y^{\prime}=J y$ when the Nordsieck representation is used.

7. New Formulas. As we have already discussed in Section 2, using the formulation (2.5), polynomial $C_{n+1}(x)$ can be obtained in terms of the two polynomials $p$ and $q$ for the second derivative formulas of Enright [3] by requiring that

$$
C_{n+1}\left(x_{n}\right)=C_{n+1}^{\prime}\left(x_{n}\right)=0
$$

and, in addition,

$$
C_{n+1}^{\prime}\left(x_{n-i}\right)=0 \text { for } i=1, \ldots, k-1
$$

for a $k$-step formula of order $k+2$. To obtain the polynomial $p$ and $q$ we require that

$$
p(-1)=q(-1)=0 \quad \text { and } \quad p^{\prime}(-i)=q^{\prime}(-i)=0, \quad i=1,2, \ldots, k,
$$

and, in addition, choosing $p^{\prime}(0)=1, p^{\prime \prime}(0)=0$ and $q^{\prime}(0)=0, q^{\prime \prime}(0)=1$.

New sets of formulas may be obtained by choosing $C_{n+1}$ to approximate zero for values $x \leqslant x_{n}$ in other ways. We have tried three different approximations and the following sets of formulas were obtained.

(1) Set 1. This set of second derivative formulas was obtained by requiring $C_{n+1}(x)$ to satisfy the following conditions:

$$
C_{n+1}\left(x_{n-i}\right)=0, \quad i=0,1, \ldots, k-1 .
$$

The above conditions lead to $k$-step formulas of order $k+1$. The polynomial $p$ and $q$ of (2.7) may be obtained by requiring that

$$
p(-i)=q(-i)=0 \text { for } i=1,2, \ldots, k,
$$


and, in addition,

$$
p^{\prime}(0)=q^{\prime \prime}(0)=1 \text { and } p^{\prime \prime}(0)=q^{\prime}(0)=0
$$

as before. These formulas could be called second derivative BDF because of the backward differentiation conditions which are satisfied by the formulas. The formulas are stiffly-stable up to order 11.

(2) Set 2 . This second set of formulas was obtained by requiring $C_{n+1}(x)$ to satisfy the following conditions

$$
C_{n+1}^{\prime}\left(x_{n}\right)=0 \text { and } C_{n+1}\left(x_{n-i}\right)=0, \quad i=0,1, \ldots, k-1 .
$$

This leads to $k$-step formulas of order $k+2$ stable up to order 8 . The polynomials $p$ and $q$ may be obtained by requiring

$$
p^{\prime}(-1)=q^{\prime}(-1)=0 \text { and } p(-i)=q(-i)=0, \quad i=1,2, \ldots, k,
$$

and the same conditions as before for $t=0$.

(3) Set 3. This set was obtained by requiring $C_{n+1}(x)$ to satisfy the following conditions

$$
C_{n+1}\left(x_{n-i}\right)=C_{n+1}^{\prime}\left(x_{n-i}\right)=0, \quad i=0,1, \ldots, k-1 .
$$

This leads to $k$-step formulas of odd order $2 k+1$. To obtain even order formulas we decided to let $C_{n+1}\left(x_{n-k+1}\right) \neq 0$. This leads to $k$-step formulas of even order $2 k$. Formulas were stable only up to order 7.

Stability curves and truncation error for the above sets of formulas were computed using the results of Sections 3 and 4. The results are summarized in Table 1 which gives the truncation error coefficients $K_{m+1}$ and stability parameters $\alpha$ and $D$ for each of the three sets of formulas described above and the formulas of [3]. For definition of stability parameters $\alpha$ and $D$, see [5, p. 219].

\begin{tabular}{|c|c|c|c|c|c|c|c|c|c|c|c|c|}
\hline \multirow{2}{*}{ ORDER } & \multicolumn{3}{|c|}{ Enright's Formulas } & \multicolumn{3}{|c|}{ Set 1} & \multicolumn{3}{|c|}{ Set 2} & \multicolumn{3}{|c|}{ Set 3} \\
\hline & $K_{m+1}$ & $\alpha$ & $D$ & $K_{m+1}$ & $\alpha$ & $D$ & $K_{m+1}$ & $\alpha$ & $D$ & $k_{m+1}$ & $\alpha$ & $D$ \\
\hline 2 & - & - & - & 0.17 & $A-S t$ & ble & 0.17 & $A-S t$ & ble & - & - & - \\
\hline 3 & $0.14 \mathrm{E}-1$ & $A$ - st & able & $0.55 \mathrm{E}-1$ & $A-S t$ & able & $0.14 \mathrm{E}-1$ & $A-S t$ & able & $0.14 \mathrm{E}-1$ & $A-S t=$ & able \\
\hline 4 & $0.49 \mathrm{E}-2$ & $A-S t$ & able & $0.27 \mathrm{E}-1$ & $A-S t$ & ble & $0.37 \mathrm{E}-2$ & $A-S t$ & able & $0.49 \mathrm{E}-2$ & $A-S t$ & able \\
\hline 5 & $0.24 \mathrm{E}-2$ & 87.9 & -0.10 & $0.16 \mathrm{E}-1$ & 89.4 & $-0.15 \mathrm{E}-1$ & $0.14 \mathrm{E}-2$ & 86.1 & -0.23 & $0.74 \mathrm{E}-3$ & 82.8 & -0.51 \\
\hline 6 & $0.14 \mathrm{E}-2$ & 82.0 & -0.53 & $0.10 \mathrm{E}-1$ & 86.4 & -0.13 & $0.67 \mathrm{E}-3$ & 77.4 & -0.96 & $0.25 \mathrm{E}-3$ & 69.7 & -1.80 \\
\hline 7 & $0.86 E-3$ & 73.1 & -1.34 & $0.73 \mathrm{E}-2$ & 80.8 & -0.40 & $0.36 \mathrm{E}-3$ & 64.4 & -2.17 & $0.51 \mathrm{E}-4$ & 46.8 & -5.53 \\
\hline 8 & $0.59 \mathrm{E}-3$ & 60.0 & -2.72 & $0.54 \mathrm{E}-2$ & 72.5 & -0.88 & $0.21 \mathrm{E}-3$ & 43.2 & -3.86 & & Unstab & le \\
\hline 9 & $0.42 \mathrm{E}-3$ & 37.7 & -5.18 & $0.41 \mathrm{E}-2$ & 60.8 & -1.65 & & Unstab & e & & & \\
\hline 10 & & Unstab & & $0.32 \mathrm{E}-2$ & 43.4 & -2.77 & & & & & & \\
\hline 11 & & & & $0.26 \mathrm{E}-2$ & 12.5 & -4.37 & & & & & & \\
\hline 12 & & & & & & & & & & & & \\
\hline
\end{tabular}

\section{TABLE 1}

Stability parameters and truncation error coefficients of four sets of second derivative formulas 
8. Conclusions. We have shown the relationship between a polynomial formulation of second derivative formulas and their conventional representation. This is useful in deriving new sets of second derivative formulas and investigating their stability. We have derived some new sets of formulas and the results are encouraging.

Also as indicated by Gupta [8], the polynomial formulation presented here could be used for computing variable-step formulas if necessary.

We have also shown that it is not at all obvious that for the problem $y^{\prime}=J y$ blended formulas of Skeel and Kong [12] are equivalent to Enright's formulas when Nordsieck representation is used.

Acknowledgement. We are grateful to an anonymous referee for a thorough review of this paper.

Appendix. Vectors of scaled derivatives of corrector polynomials $p$ and $q$ in (2.8) or the correction vectors $d$ and $e$ of (4.1) for the second derivative formulas of Enright [3] are given here. $\left(d_{i}, e_{i}\right.$ are the $i$ th elements of $d$ and $e$.)

TABLE Al

Coefficients of the correction vectors $d\left(d_{1}=1, d_{2}=0\right)$

\begin{tabular}{|c|c|c|c|c|c|c|}
\hline ORDER= & 4 & 5 & 6 & 7 & 8 & 9 \\
\hline$d_{0}$ & $\frac{29}{48}$ & $\frac{307}{540}$ & $\frac{3133}{5760}$ & $\frac{317751}{6048 i i n}$ & $\frac{247021}{483840}$ & $\frac{1758023}{3528000}$ \\
\hline$d_{3}$ & $-\frac{7}{12}$ & $-\frac{85}{108}$ & $-\frac{415}{432}$ & $-\frac{12019}{10800}$ & $-\frac{13489}{10800}$ & $-\frac{726301}{529200}$ \\
\hline$d_{4}$ & $-\frac{3}{16}$ & $-\frac{5}{12}$ & $-\frac{755}{1152}$ & $-\frac{343}{384}$ & $-\frac{16219}{14400}$ & $-\frac{9743}{7200}$ \\
\hline$d_{5}$ & & $-\frac{11}{180}$ & $-\frac{119}{720}$ & $-\frac{2149}{7200}$ & $-\frac{6503}{14400}$ & $-\frac{311821}{504000}$ \\
\hline$d_{6}$ & & & $-\frac{25}{1728}$ & $-\frac{133}{2880}$ & $-\frac{1631}{17280}$ & $-\frac{119}{756}$ \\
\hline$d_{7}$ & & & & $-\frac{137}{5040}$ & $-\frac{1009}{100800}$ & $-\frac{3069}{352800}$ \\
\hline$d_{8}$ & & & & & $-\frac{49}{115200}$ & $-\frac{179}{100800}$ \\
\hline
\end{tabular}


TABLE A2

Coefficients of the correction vectors $e\left(e_{1}=0, e_{2}=\frac{1}{2}\right)$

\begin{tabular}{|c|c|c|c|c|c|c|}
\hline ORDER= & 4 & 5 & 6 & 7 & 8 & 9 \\
\hline$e_{0}$ & $-\frac{1}{8}$ & $-\frac{19}{180}$ & $-\frac{3}{32}$ & $-\frac{863}{10080}$ & $\frac{275}{3456}$ & $-\frac{33953}{453600}$ \\
\hline$e_{3}$ & $\frac{1}{2}$ & $\frac{11}{18}$ & $\frac{25}{36}$ & $\frac{137}{180}$ & $\frac{147}{180}$ & $\frac{1089}{1260}$ \\
\hline$e_{4}$ & $\frac{1}{8}$ & $\frac{1}{4}$ & $\frac{35}{96}$ & $\frac{15}{32}$ & $\frac{203}{360}$ & $\frac{469}{720}$ \\
\hline$e_{5}$ & & $\frac{1}{30}$ & $\frac{1}{12}$ & $\frac{17}{120}$ & $\frac{147}{720}$ & $\frac{967}{3600}$ \\
\hline$e_{6}$ & & & $\frac{1}{144}$ & $\frac{1}{48}$ & $\frac{175}{4320}$ & $\frac{49}{756}$ \\
\hline$e_{7}$ & & & & $\frac{1}{840}$ & $\frac{1}{240}$ & $\frac{23}{5^{\prime}}$ \\
\hline$e_{8}$ & & & & & $\frac{1}{5760}$ & $\frac{7}{10080}$ \\
\hline$e_{9}$ & & & & & & $\frac{1}{45360}$ \\
\hline
\end{tabular}

Department of Computer Science

Monash University

Clayton, Victoria, Australia

1. C. A. AdDison, Implementing a Stiff Method Based Upon the Second Derivative Formulas, Technical Report No. 130/79, Dept. of Computer Science, University of Toronto, Canada, 1979.

2. R. L. BRown, "Some characteristics of implicit multistep multi-derivative integration formulas," SIAM J. Numer. Anal., v. 14, 1977, pp. 982-993.

3. W. H. ENRIGHT, "Second derivative multistep methods for stiff ordinary differential equations," SIAM J. Numer. Anal., v. 11, 1974, pp. 321-331.

4. W. H. EnRight, "Optimal second derivative methods for stiff systems," in Stiff Differential Systems (R. A. Willoughby, Ed.), Plenum Press, New York, 1974, pp. 95-109.

5. C. W. GEAR, Numerical Initial Value Problems in Ordinary Differential Equations, Prentice-Hall, Englewood Cliffs, N. J., 1971.

6. W. Liniger \& R. A Willoughby, "Efficient integration methods for stiff systems of ordinary differential equations," SIAM J. Numer. Anal., v. 7, 1970, pp. 47-66.

7. G. K. GuPtA, New Multistep Methods for the Solution of Ordinary Differential Equations, Ph. D. Thesis, Dept. of Computer Science, Monash University, Victoria, Australia, 1975. 
8. G. K. GUPTA, "Implementing second derivative multistep methods using the Nordsieck polynomial representation," Math. Comp., v. 32, 1978, pp. 13-18.

9. R. SACKS-DAvis, "Solution of stiff ordinary differential equations by a second derivative method," SIAM J. Numer. Anal., v. 14, 1977, pp. 1088-1100.

10. R. SACKS-DAVIS, "Fixed leading coefficient implementation of SD-Formulas for stiff ODE'," $A C M$ Trans. Math. Software, v. 6, 1980, pp. 540-562.

11. R. D. SKeEL, "Equivalent forms of multistep formulas," Math. Comp., v. 33, 1979, pp. 1229-1250.

12. R. D. SKeEl \& A. K. Kong, "Blended linear multistep methods," ACM Trans. Math. Software, v. 3, 1977, pp. 326-345 (also Report UIUCDCS-R-76-800, Dept. of Computer Science, Univ. of Illinois).

13. C. S. Wallace \& G. K. GuPTA, "General linear multistep methods to solve ordinary differential equations," Austral. Comput. J., v. 5, 1973, pp. 62-69. 